Archives

40 | 2007

Dépendance(s)

\title{
Esclavage, assimilation et dépendance
}

Essai sur une relation coloniale

\section{Myriam Cottias}

\section{(2) OpenEdition}

12 Journals

Édition électronique

URL : http://journals.openedition.org/ccrh/3394

DOI : $10.4000 /$ ccrh.3394

ISSN : $1760-7906$

Éditeur

Centre de recherches historiques - EHESS

Édition imprimée

Date de publication : 25 avril 2007

Pagination : 143-161

ISSN : 0990-9141

\section{Référence électronique}

Myriam Cottias, «Esclavage, assimilation et dépendance ", Les Cahiers du Centre de Recherches Historiques [En ligne], 40 | 2007, mis en ligne le 12 octobre 2011, consulté le 30 avril 2019. URL : http:// journals.openedition.org/ccrh/3394; DOI : 10.4000/ccrh.3394

Ce document a été généré automatiquement le 30 avril 2019.

Article L.111-1 du Code de la propriété intellectuelle. 


\title{
Esclavage, assimilation et dépendance
}

\author{
Essai sur une relation coloniale
}

\section{Myriam Cottias}

1 La notion de dépendance coloniale a été plus utilisée pour ce qui a été dénommé la « seconde colonisation ». Celle-ci débute en 1830 avec la prise d'Alger mais se développe surtout avec les conquêtes militaires européennes en Afrique et en Asie à la fin du XIX siècle. En 1920, Lénine la définissait comme un asservissement des colonies aux pays les plus riches et les plus civilisés. Presque quatre-vingts ans plus tard, le gouvernement français inscrivait dans les accords de Nouméa, ce paragraphe passé presque inaperçu :

La relation de la Nouvelle-Calédonie avec la métropole lointaine est demeurée longtemps marquée par la dépendance coloniale, c'est-à-dire un lien univoque, un refus de reconnaître les spécificités dont les populations nouvelles ont aussi souffert dans leurs aspirations.

2 Cette relation de domination d'une métropole, d'un centre, sur une périphérie, un OutreMer, est inscrite pourtant dans un processus historique qui a débuté dès le $\mathrm{XV}^{\mathrm{e}}$ siècle et comporte une caractéristique : la première expression de la colonisation européenne est en effet strictement liée à l'esclavage et à la déportation des populations d'Afrique vers les Amériques, après une expérimentation du système de plantation dans les Iles du Cap Vert, de Madère et des Açores. Pour la France, à cette caractéristique s'en est ajoutée une autre de l'ordre de la philosophie politique. Bien qu'au Xvie siècle, le mercantilisme soit la première doctrine européenne de la colonisation où la relation entre la métropole et les colonies est réglée par le "système de l'exclusif », rapidement s'y ajoute un contenu culturel. Alors qu'est affirmé par Diderot et l'Abbé Raynal, une conception universaliste de l'humanité au nom de la raison universelle qui permet d'accéder en même temps à la Vérité et au Bonheur, les philosophes construisent un paradigme européocentrique du Progrès dans une acception aussi bien matérielle qu'intellectuelle et morale. Simultanément, elle trouve une traduction et un écho dans les colonies antillaises du XVIII e siècle dans un notion protéiforme, celle de «l'assimilation » qui permet de repenser celle de « dépendance », notamment dans le contexte antillais post-abolitionniste. 


\section{« L'assimilation », une notion coloniale ${ }^{1}$}

3 En effet, la définition politique de l'assimilation s'enracine dans le débat colonial de la période de la Révolution française et s'organise selon les clivages « raciaux » admis par les sociétés coloniales des Antilles. La première occurrence du terme est attestée en 1374, selon le Trésor de la Langue Française, comme l'« action de bien intégrer un élément extérieur », tandis qu'en 1793, Condorcet, Sieyès et Duhamel y font référence comme « la première condition de l'état social $»^{2}$. Les historiographes de la nation française du XIXe siècle (Michelet, Renan, Augustin Thierry, Guizot...) l'utilisent en louant la capacité de la France à attirer, intégrer, fondre des populations différentes... sur le modèle des Romains. Pourtant, c'est aux Colonies et notamment à Saint-Domingue qu'il en a été sérieusement question sous deux formes.

4 La première concerne les citoyens « de couleur » des Colonies, c'est-à-dire des anciens esclaves affranchis. Depuis 1790 , ils la réclament afin d'obtenir l'égalité avec les autres personnes «libres", à savoir les "Blancs »3. Le préjugé de couleur élaboré au fil des siècles dans les Colonies leur conférait, en effet, une citoyenneté amputée du droit d'exercer la représentation politique ainsi que certaines professions comme la médecine, le notariat.... ${ }^{4}$ Au nom de la liberté, au nom de l'égalité de la Déclaration des Droits de l'Homme, au nom de leur richesse et à la faveur des troubles révolutionnaires, ils réclament une citoyenneté pleine et entière qui comprend, selon la Constitution de 1791, la confirmation de la nationalité5. Dès la fin des années 1789, le curé d'Emberménil réclame dans un Mémoire en faveur des gens de couleur que,

les gens de couleur de Saint-Domingue et des autres Colonies françaises, y compris les nègres libres, [soient] déclarés citoyens dans toute l'étendue du terme et en tout assimilés aux Blancs; en conséquence (qu'ils puissent) exercer tous les arts et métiers, émigrer des îles, fréquenter les écoles publiques, et aspirer à tous les emplois, ecclésiastiques, civils et militaires ${ }^{6}$.

5 Les « hommes de couleur libres » durent cependant sans cesse réclamer cette assimilation de droits ${ }^{7}$. Tout au long de la première moitié du XIXe siècle, ils revendiquent l'application des lois, et notamment celle du Code Noir : l'égalité devant la loi, l'abrogation des textes discriminatoires. Ils réclament de pouvoir hériter d'un "Blanc", de se réunir sans autorisation, de pouvoir être appelé «Sieur» et «Dame». Leur état, dénoncé dans un livret intitulé « De la situation des gens de couleur libres à la Martinique » fit d'ailleurs scandale dans la Colonie en 1823. Ses répercussions furent importantes puisqu'elles allaient entraîner des modifications législatives en faveur des « gens de couleur libres » à partir des années 1830 .

6 La seconde définition de l'assimilation émane des colons, des «Blancs» qui, en 1791, réclament que les Colonies soient des départements français d'Amérique tout en négociant une autonomie commerciale et politique des Colonies. Si leur première revendication est entérinée en 1793, la seconde reste sans résultat. En effet, après la première abolition de l'esclavage et l'arrivée d'une députation de gens de couleur, la définition de l'assimilation prend une tournure plus politique et tend à marquer les rapports de pouvoir entre la Métropole et ses Colonies. Celles-ci, portions de l'empire français, seront «essentiellement assimilées en tout, aux autres parties de la République ", selon Boissy d'Anglas, en 1795, au nom de la supériorité des peuples du Nord ${ }^{8}$. Les conditions de la relation coloniale étaient posées pour toujours : la notion 
d'« assimilation » indiquait le sens du rapport de force et de domination. Cependant, les "vieilles Colonies" continuaient à comprendre l'«assimilation" dans sa première acception, celle de «mise à égalité ». C'est d'ailleurs celle qui dominera dès lors chez les hommes politiques des Antilles, de la Guyane et de la Réunion.

7 La notion d'assimilation connaît une nouvelle étape lors de la promulgation du Code civil à la Martinique, le 7 novembre 1805. En effet, à cette occasion, les espaces de manipulation politique tout comme le spectre d'instrumentalisation de cette notion sont révélés. Presque trois ans après le rétablissement de l'esclavage, par opportunisme politique, Napoléon qui vient de subir la proclamation de l'indépendance d'Haïti et a besoin de l'appui des colonies antillaises dans la guerre contre les Anglais, conforte les colonies dans une définition racialisée de l'assimilation. Si les représentants de l'Empereur comme le Préfet Laussat la définisse comme la fusion entre les Français de l'une et l'autre rive', l'adhésion à ce « pacte d'alliance » se racialise dans l'espace colonial. Les colons dans un rapport de force qui leur est favorable, impose des changements selon «les modifications convenables $»^{10}$ et « les convenances locales».

Celles-ci s'organisent autour de deux axes :

9 - le premier veille à garantir et renforcer la barrière raciale entre «Blancs » et Affranchis dans un moment où la pression démographique des "gens de couleur» est en progression constante puisqu'ils représentent $46 \%$ de la population libre, contre $34 \%$ en 1790. Convoquant les usages coloniaux, l'article I de modification du Code civil stipule :

De tout temps, on a connu dans les colonies la distinction des couleurs, qu'elle est indispensable dans les pays à esclaves, et qu'il est nécessaire d'y maintenir la ligne de démarcation qui a toujours existé entre la classe blanche et celle des affranchis ou de leurs descendants; que cette distinction a donné lieu à plusieurs lois locales, dont le maintien et la conservation sont également nécessaires ${ }^{11}$.

10 Ainsi,

en conséquence sont maintenues toutes les lois qui ont réglé la condition des esclaves, l'état des affranchis et de leurs descendants, et la ligne de démarcation qui a toujours existé entre la classe blanche et les deux autres, ainsi que les lois faites en conséquence de cette distinction (article II).

11 - le second enjeu est celui de la propriété et plus précisément, celui de la transmission de la propriété. Ces modifications tendent là encore à garantir les barrières raciales.

Les lois du Code civil, relativement au mariage, à l'adoption, à la reconnaissance des enfants naturels, aux droits des enfants naturels dans la succession de leurs père et mère, aux libéralités faites par testaments ou donations, aux tutelles officieuses ou datives, ne seront exécutées dans la colonie, que des blancs ou blancs, entre eux, et des affranchis ou des descendants d'affranchis, entre eux, sans que, par aucune voie directe ou indirecte, aucune desdites dispositions puisse avoir lieu d'une classe à l'autre ; et seront les personnes de l'une ou l'autre classe tenues de se conformer, pour les actes de naissances, de mariage et de décès, à ce qui est prescrit par le Code civil (Article III).

12 «L'assimilation» recevait ainsi une définition ethnico-culturelle qui, aux Antilles, a été modifiée par l'ordonnance du 24 février 1831 portant abrogation des modifications du Code civil et qui n'a pas été remise en cause par les Républicains de la Troisième République en d'autres lieux. 


\section{L'assimilation et ses définitions coloniales}

13 L'assimilation avant d'être au cœur des questionnements posés dans l'hexagone français des $\mathrm{XX}^{\mathrm{e}}$ et $\mathrm{XXI}^{\mathrm{e}}$ siècles, s'est épanouie dans le milieu colonial sur un mode étrangement contemporain. La notion est récurrente dès le milieu du XIX siècle dans les traités coloniaux écrits sous le Second Empire ou la III ${ }^{\mathrm{e}}$ République mais avec des temporalités différentes selon le type de gouvernement de la France et selon les colonies.

Comme le souligne Charles-Robert Ageron, il faut noter que le Second Empire est une mise en berne de la notion d'assimilation, en particulier pour l'Algérie. Après qu'il a été dit à propos de la population indigène qu'on « était en présence d'une nationalité armée et vivace qu'il faut éteindre par l'assimilation », en 1858, Napoléon III revenait sur cette position. Il déclarait à Alger que « la France qui sympathise avec les idées de nationalité, n'a pas le droit de transformer en Français les indigènes de l'Afrique du Nord » et il demandait aux Français « le respect absolu de la mentalité, des mœurs et des situations acquises indigènes $»^{12}$.

La Restauration de la III ${ }^{\text {e }}$ épublique remit, en revanche, l'assimilation au centre des objectifs coloniaux. Dès lors, il est admis de nouveau que,

l'organisation coloniale repose sur l'union politique, sur l'assimilation, sur la centralisation. Nous tenons beaucoup à avoir nos Colonies sous la main, à notre disposition et à notre service; nous les faisons à notre image et les destinons à notre usage. [notait Jules Duval] ${ }^{13}$.

16 À propos de l'Algérie et de l'assimilation des indigènes, Antoine-Vincent Passols affirmait toujours, en 1903, que la méthode de colonisation française «s'inspirant des immortels principes humanitaires de la Déclaration des droits de l'Homme, cherche à s'assimiler les peuples vaincus [...] en les instruisant, les civilisant et les utilisant selon leurs aptitudes » ${ }^{14}$. Le discours demeurait le même chez Albert Sarraut dans un discours aux élèves de l'Ecole coloniale le 5 novembre 1923 à propos de l'assimilation :

La grande pensée de justice qui imprègne la tradition du pays de la Déclaration des droits de l'homme a repoussé le dogme cruel qui décrétait l'infériorité éternelle de certaines races. Elle constate le retard de leur évolution, mais s'employant à en corriger les effets, elle s'efforce d'en accélérer les étapes : et dans l'argile informe des multitudes primitives, elle modèle patiemment le visage d'un nouvelle humanité ${ }^{15}$.

17 Si quelques voix se sont élevées contre ce modèle assimilationniste de la colonisation dont Gustave Le Bon est le porte-parole au nom d'une phobie du métissage avec les races inférieures - elles sont restées minoritaires ${ }^{16}$.

18 L'assimilation, notion centrale de la République, a reçu cependant des définitions différentes selon les Colonies. Chaque entité coloniale a été soumise à une assimilation particulière en fonction de l'ancienneté de sa participation à l'histoire nationale, de sa maturité sociale supposée et, partant, des attributs et tâches que la Métropole lui réservait. C'est une assimilation "fonctionnaliste » qui a été mise en place selon les capacités et les qualités de civilisation que la France voulait bien reconnaitre aux personnes colonisées. Arthur Girault, principal théoricien de la législation coloniale n'allait-il pas plus loin en affirmant que la politique d'assimilation ne s'entendait que pour nos compatriotes des colonies ${ }^{17}$. Rougier, quant à lui, notait, 
on conçoit que des dispositions spéciales continuent à régir nos établissements coloniaux, à raison des éléments complexes de leur population, de leur éloignement, de leur climat et de leurs mœurs. L'assimilation à la Métropole se heurtera toujours à des différences irréductibles ${ }^{18}$.

La politique d'assimilation ne pouvait donc s'appliquer dans son intégralité qu'à certaines colonies dont les élites étaient reconnues par la Métropole et qui, elles-même, s'appropriaient les standards culturels de la Métropole. Ainsi, dès le senatus-consulte du 7 avril 1854, l'article 18 stipule-t-il que «les colonies autres que la Martinique, la Guadeloupe et la Réunion seront régies par décrets de l'Empereur jusqu'à ce qu'il ait été statué à leur égard par un sénatus-consulte ». Ainsi, dans l'ensemble colonial, le Sénégal n'était-il pas traité comme l'Inde; la Martinique et la Guadeloupe n'étaient pas considérées de la même façon que la Guyane... Cette colonie est soumise, en effet, à la fin du xIXe siècle, à un régime semblable à celui du Sénégal. Dans ce cortège de différences entre les Colonies, on peut noter que le décret du 24 février 1885, portant sur les conseils généraux indique qu'au Sénégal et seulement là, « les conseillers généraux doivent savoir parler, lire et écrire le français $»^{19}$.

Si les «vieilles Colonies » ont persisté à revendiquer l'assimilation comme " égalité des personnes et des droits » avec la Métropole; celle-ci, en revanche, les a considérées dès 1852, dans le cadre d'une relation coloniale qu'elle pouvait modifier à son seul gré. En dépit d'acquis fondamentaux garantis et d'une relation particulière à l'État, les métamorphoses institutionnelles le prouvent. La question du statut législatif de ces territoires en est un exemple, entre autres. En effet, si la Constitution du 4 novembre 1848 déclarait les Colonies, territoires français, celle de janvier 1852, leur impose un statut d'exception en leur enlevant leur représentation parlementaire et en les régissant par sénatus-consulte. En dépit du rétablissement de la représentation coloniale à l'Assemblée nationale en 1871, c'est pourtant le gouvernement qui, par décret, étendait aux Colonies le bénéfice des lois métropolitaines. Après 1880 , ces dispositions furent de nouveau modifiées et il fut institué que les lois étaient uniquement promulguées dans les Colonies sur mention expresse. Les lois métropolitaines n'étaient donc pas applicables de plein droit aux Colonies ${ }^{20}$ et cela a perduré jusqu'à la Constitution du 27 octobre $1946^{21}$. La loi du 19 mars 1946 sur « le classement comme départements français de la Guadeloupe, de la Martinique, de la Réunion et de la Guyane française » maintenait, en effet, à titre transitoire la spécialité législative. Au niveau national comme local, elle fut appelée dès sa promulgation « loi d'assimilation » malgré la réfutation de ce terme par Aimé Césaire le 4 mai 1948 qui proposait de la désigner comme « loi de départementalisation $»^{22}$.

\section{L'assimilation, renfort de la dépendance en situation post-abolitionniste}

21 L'abolition de l'esclavage décrétée le 27 avril 1848 par le Gouvernement Provisoire de la Seconde République ${ }^{23}$ remodelait l'espace public dans les Colonies françaises par l'instauration de l'égalité entre les citoyens. À tous les affranchis et à tous ceux qui y étaient nés ou qui y résidaient depuis six ans ${ }^{24}$, le gouvernement attribuait la citoyenneté française par la circulaire ministérielle du 7 mai $1848^{25}$ : « à partir du jour de la libération générale, les esclaves deviendront des citoyens français ${ }^{26}$ afin qu'aucune exception au principe de liberté et de l'égalité sociale, ne puisse exister ${ }^{27}$. La portée de la décision politique prise par le Gouvernement Provisoire était immense. Non seulement, elle 
établissait une citoyenneté similaire pour des acteurs depuis toujours opposés sur le plan civil, en les intégrant à la mère-patrie, mais elle semblait renverser les relations de domination au nom de la « fusion sociale ». Les horizons sociaux et mentaux des anciens affranchis comme des anciens maîtres devaient s'ouvrir les uns aux autres car «pour les membres d'une même nation, il n'existe qu'un seul droit, le droit commun », précisait le commissaire-général, Perrinon, le 5 juin 1848, à son arrivée à la Martinique.

En 1848, le Droit commun est donc introduit comme mécanisme de régulation des rapports sociaux. La République pouvait-elle cependant réussir à transformer les formes de domination? Le nouveau contrat social instauré par la Seconde République établissait l'égalité, l'assimilation des citoyens coloniaux antillais et réunionnais à la Métropole. La «justice politique » telle que définie par John Rawls ${ }^{28}$ et discutée par Jean-Marc Ferry ${ }^{29}$ comme le fait que, «chaque personne doit avoir un droit égal au système le plus étendu de libertés de base égales pour tous, qui soit compatible avec le même système pour tous les autres ", sous-tendait la pensée philosophique des hommes politiques de la Seconde République. Cette égalité républicaine comprenait des «droits de citoyenneté30 qui, pour les Colonies, s'appuyaient sur l'effacement des traces serviles marquant l'identité juridique et sociale de plus de la moitié de la population coloniale. L'individualité par collation de noms patronymiques est donc affirmée comme base du nouveau contrat social posé par la Seconde République ${ }^{31}$. Dans une séance du mois de juin 1848 ,

Le commissaire-général de la République spécifie que les noms patronymiques des nouveaux libres figureront sur leur titre de liberté avec lesquels on leur délivrera un exemplaire du décret du 27 avril portant abolition de l'esclavage. Le préalable à tout établissement du droit était, en effet, de pouvoir identifier, reconnaître à chacun une individualité qui lui permettait, en retour, d'acter le droit. Dans cette relation de réciprocité établie alors, chaque individu était à la fois sujet du droit (en tant que récepteur des lois) et acteur du droit (en tant qu'il pouvait saisir la justice) ${ }^{32}$.

Dès la promulgation officielle de l'émancipation à l'arrivée du commissaire-général de la République à la Martinique, comme à la Guadeloupe, la question de l'attribution d'un patronyme est posée. En effet, dans la mise en œuvre de l'État de droit, elle constitue un problème pratique : d'une part, il est nécessaire d'établir la base des citoyens actifs, c'està-dire des hommes, appelés à voter grâce à l'instauration de l'égalité civile et politique avec la Métropole; de l'autre, ces derniers étaient aussi appelés à participer aux nouvelles formes de juridictions de conciliation instaurées par la République sous la forme des jurys cantonaux.

Les modes d'appropriation de ce «droit commun » issu de l'acte d'Émancipation et de l'élaboration d'une société égalitaire étaient multiples. Selon les acteurs, certains actes administratifs faisaient sens dans la terminologie de Max Weber ${ }^{33}$, d'autres pas. Si les anciens maîtres comme les anciens libres de couleur, affranchis avant 1848 , enfermaient en partie le terme dans un cadre légaliste, plus ou moins rigide, les "nouveaux libres " dessinaient par leurs revendications, notamment dans les situations de conflit, leur propre définition du droit. En cette période d'ajustement des rapports sociaux, deux conceptions s'affrontaient. Elles reprenaient cependant les anciennes lignes de fractures civiles car les émissaires de la République encourageaient la prééminence du droit positif. 


\section{Droit par le travail et vertus citoyennes}

Dans un souci de stabilité sociale et économique, les Commissaires de la République se sont attachés à informer la population nouvellement affranchie de ses droits et de ses devoirs en des termes qui rendaient ces deux notions équivalentes. S'ils confirmaient que les cultivateurs étaient devenus citoyens français et qu'à ce titre, ils en avaient tous les droits, ceux-ci n'étaient pas détaillés. En revanche, leurs devoirs dont «le premier et le plus saint de tous est le travail » étaient largement commentés. La figure du «Bon citoyen " brossée depuis le début des campagnes abolitionnistes ${ }^{34}$ est alors consolidée. Elle renvoie à trois valeurs congruentes avec le contexte de moralisation générale de la société française : la « Famille », patriarcale, la « Propriété » et surtout le « Travail » dont la fonction curative permettait à la classe laborieuse de mériter la liberté.

Pourtant, dès juillet 1848, un glissement conceptuel se produit. L'Émancipation par la Seconde République ouvrait l'accès au droit commun qui comprenait un droit au travail dont l'État devait assurer l'accomplissement grâce, par exemple, à la création des Ateliers Nationaux, créés dans la Métropole au nom de la fraternité pour résoudre le chômage des ouvriers mais immédiatement interprétée aux Antilles comme une nouvelle mesure de coercition. Rapidement, au droit au travail se substitue le droit par le travail (le second terme donnant accès au second) qui trouve en 1852 son point d'orgue avec un arrêté du Directeur de l'Intérieur qui promulgue dans les Colonies le décret napoléonien sur le régime des livrets et le vagabondage $e^{35}$.

Le droit au travail, devoir de l'État vis-à-vis du citoyen, s'est rapidement métamorphosé pour le citoyen colonial nouvellement affranchi, en devoir de travailler. Sous la forme d'«associations » qui n'avaient de fouriériste que le nom ${ }^{36}$, anciens maîtres et anciens esclaves se sont liés par des contrats, favorables aux premiers. Bien que, dès le mois de juin 1848, Perrinon ait rédigé des modèles de contrats d'association qui se voulaient équitables ${ }^{37}$, ceux-ci ont été à chaque fois interprétés à l'avantage des propriétaires, notamment en cas d'absence non justifiée des travailleurs. Si Perrinon prévoyait que « chaque associé recevra une part proportionnée au nombre des journées de travail qu'il aura fourni à la société », Pierre Dessalles, lui, inscrit dans son contrat d'association que les manquements au travail non agréés devront être payés, en numéraire, du double de la valeur d'une journée de travail. Par ailleurs, les journées de travail sont de neuf heures ; les samedis, dimanches et jours de fête non ouvrés. Le propriétaire doit attribuer un jardin et une case à chaque travailleur (homme et femme) tant qu'il est dans le contrat d'association; le travailleur a droit au tiers brut ou par moitié des sucres, "délivrés en nature au fur et à mesure de la fabrication $»^{38}$. Le propriétaire, lui, fournit les terres de l'habitation, les bâtiments d'exploitation, les cases des travailleurs, les ustensiles et les instruments aratoires ${ }^{39}$. Il a, à sa charge, les frais de médecin et de médicaments ainsi que ceux dus à des blessures reçues pendant la journée de travail. $60 \%$ environ des travailleurs ruraux de la Martinique en 1848 sont engagés dans ce type de contrat ${ }^{40}$.

\section{Instances de conciliation et dépendance}

Pour résorber les tensions qui existaient sur les plantations et atteindre un accord mutuel entre les propriétaires et les travailleurs, Victor Schoelcher, président de la Commission 
d'abolition de l'esclavage, avait préconisé l'institution de jurys cantonaux, finalement décrétée le 27 avril 1848 par le Gouvernement provisoire :

Aux colonies où l'esclavage est aboli par décret de ce jour, il sera établi dans chaque ressort de justice de paix, un jury composé de six membres siégeant, en audience publique, au chef-lieu de canton, sous la présidence du juge de paix. [article ${ }^{\mathrm{er}}$ ].

31 Sur le modèle des prud'hommes institués le 18 mars 1806 à Lyon pour trancher les petits différends nés quotidiennement dans l'industrie de la soie, les jurys cantonaux réunissaient à égalitée ${ }^{41}$ "les citoyens qui possèderont ou exerceront une industrie, et les travailleurs industriels et agricoles». Ils sont désignés par tirage au sort des hommes inscrits sur les listes électorales sous condition d'âge, de nationalité et de résidence, ont obligation de siéger deux fois par semaine et reçoivent une indemnité de $2 \mathrm{~F}$ par chaque jour de séance.

32 Les jurys cantonaux possédaient une double attribution puisqu'ils avaient compétence en matière civile sur toutes contestations sur l'exécution des engagements (art. 5) et, en matière pénale, sur «tout fait tendant à troubler l'ordre et le travail dans les ateliers, chantiers, fabriques ou magasins, tous manquements graves des propriétaires ou chefs d'industrie et des ouvriers ou travailleurs, les uns envers les autres » (art 7) ${ }^{42}$. Organe de gestion des conflits journaliers, cette institution avait pour objectif de résoudre les antagonismes par la conciliation, au sein d'une instance de proximité, plus accessible à des acteurs sociaux peu habitués au droit positif et souvent incapables de s'exprimer en français. Recueillant les doléances, des uns et des autres, les jurys cantonaux procédaient d'une justice de l'oralitét ${ }^{43}$, dont des traces n'ont été conservées que dans des rapports administratifs et dans des mentions personnelles de propriétaire.

Formellement, le nouvel ordre égalitariste donnait aux individus la possibilité de constituer leurs intérêts individuels et collectifs et de les faire reconnaître comme tels ${ }^{44}$, notamment en matière civile. Traitant des litiges de travail, ces affaires pointaient, en creux, les récriminations des propriétaires et les aspirations des populations nouvellement affranchies qui se sont portées majoritairement demandeurs dès l'instauration de la juridiction cantonale. Au cours de la première année de civilité, à la Trinité, ils portent plainte dans $57 \%$ des affaires, puis, entre 1848 et 1850 , dans $60 \%$ des cas enregistrés à la Trinité, $70 \%$ au Fort-de-Saint-Pierre et $61,5 \%$ au Mouillage ${ }^{45}$. Au total, dans cinq cantons sur huit à la Martinique, les demandes émanent principalement des travailleurs ${ }^{46}$.

Leurs demandes révèlent leur expérience sociale du travail et les modifications qu'ils en attendent ${ }^{47}$. Elles se résument, pour $76 \%$ d'entre elles à la Trinité, en une demande de salaire et, dans d'autres cantons, à la jouissance de la case et des jardins qui leur permettrait à leurs yeux de rompre la relation de subordination à l'ancien maitre. Aux audiences, ils viennent donc demander, le plus souvent en groupe, cette régulation des rapports sociaux comme élément essentiel à leur statut de citoyen libre ${ }^{48}$.

La notion de "confiance » et son contraire la "défiance ", expliquent à elles seules la plupart des affaires jugées à la demande des cultivateurs. L'association qui avait la préférence tant $\mathrm{du}$ Gouvernement provisoire que des propriétaires (après quelques hésitations) obligeait à un maintien de relations organisées comme au temps de l'esclavage. En dehors de l'élection d'un conseil de prud'hommes servant d'intermédiaire entre le propriétaire et les travailleurs, de la signature conjointe d'un contrat passé devant notaire (éventuellement), le procès de travail non seulement n'avait pas changé mais, en plus, ne garantissait pas la propriété de la case et des jardins traditionnels. Or, se 
demandaient les travailleurs devant les tribunaux, comment croire à un partage équitable et garanti des productions au tiers ou au quart, quand le rapport d'échange paraissait si inégal, et quand l'économie vivrière élaborée pendant l'esclavage était menacée par le droit de propriété des anciens maitres. Des récoltes de manioc, de bananes, plantées avant l'Abolition ou avant la signature des actes d'association, étaient réclamées par les cultivateurs. Les discussions dérisoires mais symboliquement fortes, s'éternisaient pour savoir si les fruits pendants aux arbres appartenaient à l'ancien maître ou aux nouveaux affranchis ${ }^{49}$ tandis que d'autres devaient décider si un travailleur marié pouvait recevoir sa femme dans sa case quand celle-ci ne participait pas au contrat d'association passé avec le maitre...

Face aux aspirations sociales des nouveaux affranchis, les propriétaires, eux, venaient chercher confirmation, en matière civile, de leur droit de propriété et de leur position d'autorité dans ce nouveau cadre juridique. Dénonçant l'abandon du travail par les travailleurs ( $36 \%$ des demandes), la mauvaise exécution des contrats (27\%), ils demandent leur expulsion dans $50 \%$ des demandes. Il ne semble pas que le tribunal leur ait demandé de préciser des plaintes apportées sans démonstration de preuves - au contraire de celle portée contre Bastienne à qui il est reproché « d'avoir perdu 33 jours de travail sur $56 \Perp^{50}-$. Or, le plus souvent les plaintes des propriétaires résultaient d'une histoire longue où les travailleurs essayaient de faire entendre leurs attentes. Le 5 mai 1849, La Disette et d'autres cultivateurs de Pierre Dessalles sont jugés pour « abandon du travail » mais la description du conflit par le propriétaire révèle tout autre chose. Depuis des semaines, ils sont mécontents du prix des sucres qui leur ont été distribués, aspirent à un partage des terres (qui devaient se faire par l'intermédiaire de Cyrille Bissette, (" homme de couleur ", abolitionniste) et, enfin, veulent la confirmation de la propriété de leurs cases. En regard, le propriétaire se plaint continûment de la lenteur du travail mais souligne au lendemain de l'altercation violente qui provoque l'assignation de l'atelier, que « les nègres se sont montrés au travail $»^{51}$ : l'accusation portée devant le jury cantonal était donc fausse. Résumer le conflit ainsi permettait, en revanche, d'atteindre deux objectifs: d'une part, d'être entendu par les juges de paix chargés par le Gouvernement de garantir la stabilité économique; de l'autre, de rétablir leur rapport d'autorité en obligeant les nouveaux affranchis à accepter les conditions de vie et de travail.

\section{Les affaires pénales ou le maintien de la dépendance}

Les affaires pénales illustrent encore plus cette utilisation du droit. Elles rassemblaient tous les conflits qui menaçaient directement la relation d'autorité et pouvaient engendrer ce qui était considéré comme de la désorganisation sociale. Défini par l'article 7 du décret, il s'agissait des disputes, du manque de respect, de la désobéissance, des paroles grossières, des injures verbales et autres faits de même nature ${ }^{52}$. Sous ce chapitre, 61 affaires sont jugées, à la Martinique, entre 1848 et 1850, toutes sur demande des propriétaires relayées par le Ministère Public, une en Guyane pendant les six premiers mois de 1850, à Cayenne, aucune en Guadeloupe.

Comme en matière pénale, les plaintes des maitres reposaient sur des histoires longues résumées pour la majorité d'entre elles, devant le jury par l'accusation de «manquements graves » et d'«insultes ». La banalité des faits reprochés peut surprendre car la violence de la servitude a toujours été accompagnée d'expressions brutales de déconsidération de 
tous les individus (dont seulement les termes les plus acceptables ont été retranscrits dans les textes) qui ont beaucoup marqué la mémoire des nouveaux affranchis d'après les rapports administratifs :

J'ai principalement remarqué, parmi les nouveaux libres, l'oubli des coups et de la rigueur du régime qui leur était précédemment appliqué, mais le souvenir vif et complet des injures qu'ils ont reçues et des injustices dont ils ont été victimes ${ }^{53}$.

Si les esclaves/nouveaux affranchis étaient traités d'« imbéciles » ou de « Nègres » (ce qui équivalait à les traiter d'esclaves), les maîtres/propriétaires, eux, sont des «voleurs » et des « assassins ». L'«insolence » dénoncée par le maître pendant l'esclavage a continué à caractériser la manifestation des idées, des opinions, des souhaits, voire de l'opposition des cultivateurs mais les modalités d'affirmation de la hiérarchie sociale avaient changé. Avec l'Émancipation, la parole de l'affranchi était formellement mise à égalité avec celle du maître. Alors que pendant l'esclavage, le maitre avait les moyens d'endiguer toute contestation, aussi minime fût-elle (car "c'est moi le maître ", écrit Dessalles le $1^{\mathrm{er}}$ janvier 1839) par le fouet ou les piquets ${ }^{54}$, après l'Émancipation, le différend devait être médiatisé par la loi.

La convocation devant le jury cantonal était donc la conséquence d'un long processus de contestation qui avait les mêmes motifs que les conflits jugés en matière civile : propriété des terres et des cases, taille des jardins, partage des sucres. Pourtant, un autre élément s'y mêlait : celui de la verbalisation de la contestation de l'autorité. Refuser un économe qui ne convenait pas à un atelier, des pratiques qui évoquaient l'esclavage ${ }^{55}$, des ordres jugés excessifs frôlait la révolte aux yeux des propriétaires et s'exprimait en « cris et en propos injurieux", en menaces "de voir bientôt jouer le coutelas", en scènes paroxystiques où les travailleurs parodiaient les corrections par le fouet... C'était cette mise en cause extrême de leur pouvoir que les propriétaires portaient à l'arbitrage du jury. Les acteurs du conflit avaient aussi leur importance. Deux catégories de travailleurs étaient assignées. Elles regroupaient ceux qui ayant déjà fait bouger les cloisonnements sociaux de la dépendance, pouvaient avoir accès à la parole.

Les femmes ont été assez nombreuses à être appelées à comparaitre. Alors qu'elles constituent entre la moitié et les deux tiers des ateliers, elles forment $35 \%$ des individus cités par des propriétaires avec qui elles ont des relations particulières. Sur l'habitation de Pierre Dessalles, elles s'appellent Man, Joséphine ou encore Bastienne et ont un positionnement privilégié dans des relations intermédiaires entre le groupe des maîtres/ propriétaires et celui des travailleurs. Joséphine est connue pour ces insolences à répétition, au point que le maître la croit folle tandis que Man a participé aux stratégies sexuelles mises en place par certaines femmes pour trouver une solution personnelle aux maux de l'esclavage ${ }^{56}$. Pendant quelque temps, elle a vécu avec l'ancien économe et en tire un privilège qu'elle affich ${ }^{57}$ : la dépendance avait une dimension sexuelle. Dans un ordre colonial où le statut civil et « racial » déterminait les droits et les devoirs de chacun, l'utilisation de la sexualité donnait aux femmes la possibilité de transgresser cet ordre, notamment en leur permettant de s'exprimer plus ouvertement.

L'accès à la parole, aux insultes, aux « manquements » et aux menaces était facilité pour une deuxième catégorie de travailleurs: celle des ouvriers spécialisés ou des représentants des prud'hommes de l'habitation. L'histoire de Césaire est à ce sujet édifiante. Né sur l'habitation, il a toujours eu la confiance du maître pendant la période de l'esclavage, que ce soit sur le plan du travail ou comme intermédiaire entre maitres et femmes esclaves, et cette position privilégiée l'a conduit à être le représentant des 
travailleurs en tant que prud'homme de l'habitation. Il s'y est marié le 11 janvier 1840 et a bonne réputation. Pourtant dès l'Émancipation, il se trouve à la tête du mouvement de revendications qui va le conduire devant le jury cantonal par deux fois. Le 9 octobre 1848, en particulier, c'est lui qui, après avoir discuté la taille des jardins, après avoir refusé que les absences des travailleurs soient marquées, après avoir demandé le départ de l'économe, les engage à cesser le travail. Il est condamné pour cela à $40 \mathrm{~F}$ d'amende.

Devant les jurys cantonaux, deux pratiques et deux logiques s'affrontaient donc. Pour les travailleurs, il s'agissait de venir faire entendre leurs aspirations et donc de réclamer de l'égalité tandis que les maîtres passaient par cette instance pour rétablir un rapport de force favorable et ainsi maintenir les nouveaux affranchis dans la dépendance.

\section{«Les travailleurs auront toujours tort, les propriétaires toujours raison » : la force du préjugé}

Un an après leur création, les rapports des magistrats délégués à l'inspection des jurys cantonaux de la Martinique ${ }^{58}$, le 28 mai 1849 , et de la Guyane insistent sur les dysfonctionnements de l'institution. Ils soulignent que les listes électorales sont constituées sur des bases qui ne sont pas rigoureuses : elles sont mal tenues et ne portent souvent pas le nom patronymique des nouveaux affranchis, ni même l'exactitude quant aux professions des citoyens. Dans la plupart des bourgs, il n'y a donc qu'une seule urne, rassemblant les deux catégories de citoyens appelés à siéger, parfois ouverte aux quatre vents et suscitant la fraude. Les notifications administratives de convocation au tribunal n'arrivent pas non plus aux intéressés et, par suite, «le tribunal est arbitrairement composé et n'offre plus aucune garantie d'impartialité $\aleph^{59}$. À la Trinité, aucune session ne respecte les règles de parité sociale, sur l'ensemble de la période. Les propriétaires sont généralement plus nombreux à siéger que les cultivateurs et face aux premiers, les seconds prennent la parole timidement dans un créole traduit au juge de paix par des propriétaires ou des artisans. L'inégalité de condition sociale ne pouvait être oubliée.

Les condamnations marquaient, elles, les limites des transformations de l'espace social et le maintien d'un préjugé d'ordre " racial ». Aux maîtres étaient associés la " civilisation ", aux esclaves, l'obscurantisme. Même si ce retard était attribué par les abolitionnistes au système de l'esclavage, il n'en demeurait pas moins vrai qu'il fallait, selon eux, endiguer et contrôler les pulsions des populations appelées à la Liberté.

Perrinon ne disait-il pas,

que l'autorité doit leur expliquer et leur faire observer la loi, afin de protéger la liberté de tous ; qu'à la suite d'une transformation sociale aussi profonde, elle ne saurait abandonner les propriétaires aux seules chances de l'action civile que tout citoyen peut entamer ${ }^{60}$.

47 En dépit de l'égalitarisme républicain, leur accès au droit devait être contrôlé et la capacité d'appréciation des travailleurs de leur «bon droit» ne leur était pas reconnue par les agents de l'État.

Au nom de la propriété et de l'équilibre, les Républicains ont maintenu la hiérarchie sociale des Colonies. Plus du tiers des cultivateurs est débouté de ses demandes tandis qu'aucun propriétaire ne l'est. En matière civile, la demande de salaire n'est jamais satisfaite alors qu'en matière pénale, toutes les demandes d'expulsion le sont. Singulièrement, la conciliation n'est obtenue qu'une seule fois. $29 \%$ des propriétaires 
sont condamnés à des contraventions allant de 95 centimes à $99 \mathrm{~F} 05$; tandis que celles des cultivateurs vont de $8 \mathrm{~F}$ (pour une seule personne) à $13000 \mathrm{~F}$ pour tout un atelier. La journée de travail étant estimée à $1 \mathrm{~F}$ pour les adultes et 50 centimes pour les enfants, ces peines étaient inapplicables et ont provoqué l'évanouissement des condamnés dans les campagnes martiniquaises, relatent les rapports. Il faut noter cependant qu'une fois leur position d'autorité confirmée par les jurys cantonaux, les relations entre maîtres et travailleurs ont continué sur le même mode. Quelques temps après leur condamnation, les travailleurs de Pierre Dessalles demandent à revenir dans l'association, ce que le propriétaire accepte toujours. À tout le plus, le maître donne-t-il une journée de congé et attend-il que les travailleurs soient repentants. La vie normative se remettait en place.

Dans cette situation, seul le sentiment d'injustice - comme impossibilité d'avoir les moyens de faire reconnaître ses attentes - dominait provoquant des accès de rage impuissante comme celui de ce cultivateur siégeant à Fort-de-France qui s'écriait en pleine session : « les travailleurs auront toujours tort, les propriétaires toujours raison ». À cause de cette défiance des nouveaux affranchis qui s'est élaborée petit à petit, la transformation de l'espace social s'est accompagnée de luttes, sans résonance au niveau des jurys cantonaux. Entre le deuxième trimestre 1848 et le quatrième trimestre 1850, 348 jugements sont jugés à la Martinique (pour une population de 122000 personnes au sein de laquelle $60 \%$ étaient esclaves en 1847). Ce nombre a chuté jusqu'à devenir nul en 1850. $65,8 \%$ des affaires sont jugées au cours de la première année de civilité ${ }^{61}$. Les administrateurs en concluaient que «les rapports entre les propriétaires et les travailleurs ne donnent lieu qu'à de rares contestations. Celles-ci alors qu'elles surgissent sont le plus souvent réglées par l'intermédiaire officieux d'influences salutaires ${ }^{62}$.

Les jurys cantonaux n'ont donc bouleversé que furtivement et marginalement la grammaire de la dépendance. L'espace public était finalement réduit aux anciennes relations d'assujettissement. Entre 1848 et 1850, il y a bien eu faillite de la reconversion des rapports de domination par l'exercice de la légalité dans une instance de proximité, engendrant une défiance tenace vis-à-vis de la Loi et une incrédulité permanente par rapport au Droit. En effet, la distanciation et la médiatisation entre l'habitation et la sphère publique par des agents neutres n'avaient pas été possibles au sein des jurys cantonaux: les revendications des nouveaux affranchis, non seulement avaient été déboutées, mais aussi rendues illégitimes par les représentants de l'État. Les «nouveaux affranchis » étaient renvoyés à leur état d'«anciens esclaves » ${ }^{63}$ avec l'appui d'un Droit, égalitariste dans son essence.

\section{NOTES}

1. Voir Myriam Cottias, «Le silence de la nation. Les "vieilles colonies" comme lieu de définition des dogmes républicains ", Outre-Mers, Paris, 2003, p. 90, p. 338-339.

2. Emmanuel-Joseph Sieyès, Journal d'Instruction sociale (Paris: 1793), p. 146.

3. Des guillemets sont mis autour des caractérisations de couleur des individus pour indiquer qu'elles sont des catégories socialement et politiquement construites. Florence Gauthier, «Le 
Rôle de la députation de Saint-Domingue dans l'abolition de l'esclavage [...] » in Les Abolitions de l'esclavage, Marcel Dorigny (éd.), Paris, Presses Universitaires de Vincennes et Éditions UNESCO, 1995, p. 199-211.

4. Une étude précise de cette question a été faite par Jean-Luc Bonniol, La Couleur comme maléfice, Paris, Albin Michel, 1992.

5. Patrick Weil, Qu'est-ce qu'un Français? Histoire de la nationalité française depuis La Révolution, Paris, Grasset, 2002. p. 20.

6. Cité par Pierre Pluchon, Histoire de la colonisation française, I vol., Paris, Fayard, 1991.

7. Voir le chapitre "Assimilation politique des nouveaux citoyens" in Laurent Dubois, Les Esclaves de la République. L'histoire oubliée de la première Émancipation, 1789-1794, Paris, CalmannLévy, 1998.

8. Florence Gauthier, «Le Rôle de la députation de Saint-Domingue dans l'abolition de l'esclavage ", op. cit., p. 210.

9. «Vos cœurs palpiteront toujours au cri de la Mère-Patrie ; et les français de l'une à l'autre rive ne cesseront de se tenir fraternellement embrassés, malgré les mers vastes, et souvent ennemies, qui les séparent ", assurait Laussat, Préfet colonial devant la Cour d'appel de la Martinique, le 9 novembre 1805.

10. Procès-Verbal de la séance de La Cour d'Appel de La Martinique, relativement à la promulgation du Code Civil, Bulletin Officiel de la Martinique, Fort-de-France.

11. Arrêté colonial, concernant la promulgation du Code Civil à La Martinique, Bulletin Officiel de la Martinique, Fort-de-France.

12. Charles-Robert Ageron, France coloniale ou parti colonial ? Paris, Presses Universitaires de France, 1978.

13. Jules Duval, L'Algérie et les colonies françaises,. Paris, Guillaumin, 1877, p. 296.

14. Antoine-Vincent Passols, L'Algérie et l'assimilation des Indigènes musulmans, Paris, H. CharlesLavauzelle, 1903). p. 14 ; Fanny Colonna, "Educating Conformity in French Colonial Algeria ", Tensions of Empire . Colonial Cultures in a Bourgeois World, Frederick Cooper, Stoler, Ann Laura, (eds), Berkeley Los Angeles, London, University of California Press, 1997.

15. Alain Ruscio, Le Credo de l'homme blanc, Bruxelles, [Paris], Éd.Complexe, 1996, p. 99, cité par Claude Liauzu, La Société française face au racisme, Bruxelles, Éd. Complexe, 1999.

16. Pierre-André Taguieff, La force du préjugé. Essai sur le racisme et ses doubles, Paris, La Découverte, 1988.

17. Charles-Robert Ageron, France coloniale ou parti colonial ? op. cit.

18. J.-C. Rougier, Précis de législation et d'économie coloniale, Paris, Larose, 1895. p. 77.

19. Alain Chantre-Grellet, Traité des élections, Paris, P. Dupont, 1898. p. 299.

20. J.-C. Rougier, Précis de législation [...]., op. cit.,p. 84.

21. Article 73 : «Le régime législatif des départements d'outre-mer est le même que celui des départements métropolitains ».

22. Communication d'Emmanuel Jos, professeur de droit à l'Université des Antilles et de la Guyane.

23. Promulgué le 23 mai pour la Martinique, le 27 mai pour la Guadeloupe et le 10 août pour la Guyane après le soulèvement des ateliers d'esclaves de la Martinique.

24. Il est intéressant de noter que «le 28 mars 1848, le gouvernement provisoire de la République prend un décret autorisant temporairement le ministre de la Justice à accorder la naturalisation à tous les étrangers qui résident en France depuis cinq ans au moins ", Patrick Weil , Qu'est-ce qu'un Français? [...], op.cit.,. p.44. Voir également démographiques, M.d.f.S.n.d.s.D.d.l.s.g.E. (1942) Les naturalisations en France (1870-1940) (Paris, Imprimerie nationale).

25. Portant instructions pour l'exécution du décret du 27 avril 1848. 
26. Le terme de "citoyenneté française» indissociablement lié comprenait d'une part, la condition de nationalité et, d'autre part, l'exercice des droits citoyens des individus dans l'acception de la Seconde République, il n'est donc pas de débat qui tienne sur le fait que la nationalité des anciens esclaves ne soit pas affirmée. Anne Girollet, Victor Schoelcher, abolitionniste et républicain. Approche juridique et politique d'un fondateur de la République. Paris, Karthala. 2000.

27. Réciproquement, la citoyenneté française est incompatible avec le fait de posséder des esclaves : « il est interdit à tout Français de posséder, d'acheter ou de vendre des esclaves, et de participer, soit directement, soit indirectement, à tout trafic ou exploitation de ce genre. Toute infraction à ces dispositions entraînera la perte de la qualité de français », précise le décret d'abolition de l'esclavage. L'équivalence entre liberté, d'une part, nationalité et citoyenneté, d'autre part, était donc rendue effective et exécutoire immédiatement. Article 8 du décret relatif à l'abolition de l'esclavage et à l'organisation de la liberté, Bulletin officiel de la Martinique, 1848.

28. John Rawls, Théorie de la Justice (Paris, Seuil, 1987). Jean-Marc Ferry, Philosophie de la communication. Justice politique et démocratie procédurale, Paris, Les éditions du Cerf, 1994., vol. 2, p. 14.

29. Jean-Marc Ferry,.ibid., p. 14.

30. Jean-Marc Ferry,.op. cit.

31. «Du reste, il sera indispensable de faire procéder par les officiers de l'état civil à un enregistrement général de la population émancipée, en prenant pour point de départ les registres matricules existants et en conférant des noms aux individus et aux familles comme on l'a fait jusqu'à ce jour dans le système de l'affranchissement partiel, conformément à une ordonnance du 29 avril 1836 », Circulaire ministérielle du 7 mai 1848 portant instructions pour l'exécution du décret du 27 avril 1848.

32. L'attribution d'un nom permettait aussi l'accession à un état civil réservé auparavant aux seuls libres. S'il est des débats pour savoir si les actes d'état civil doivent être délivrés individuellement ou par famille, il y en eut peu pour accepter que ces actes soient payants au bénéfice des officiers municipaux.

33. Max Weber, Économie et Société, Paris, Plon., 1971, p. 4.

34. Sur cette période voir Y. Debbasch, (1977) «Le rapport au travail dans les projets d'affranchisse-ment: l'exemple français (XVIII'-XIXe siècles) ", Actes du XLII congrès international des Américanistes p. 203-222; Myriam Cottias, «Droit, justice et dépendance dans les Antilles françaises (1848-1852) », Annales Histoire, Sciences Sociales, Paris, Armand Colin, 2004, mai-juin, 3, p. 547-567.

35. Bulletin Officiel de la Martinique, 9 octobre 1852. Ce texte est complété le 20 mai 1854 par un arrêté du Gouverneur de la Martinique, le Comte de Gueydon.

36. Lawrence C. Jennings, French Anti-Slavery. The Movement for the Abolition of Slavery in France, 1802-1848, Cambridge, Cambridge University Press, 2000. Voir la discussion de la conception de l'association de Louis Blanc dans l'Atelier. Organe spécial de la classe laborieuse, octobre 1847. Et plus encore le contrat d'association ouvrière publié dans le même volume dont les termes sont fort éloignés du modèle proposé par Perrinon.

37. «J'ai été généralement compris, les conventions ont été immédiatement faites sur la base d'un contrat modèle d'association que j'avais pu établir pour le répandre dans la colonie [...]. Jusqu'à présent, c'est l'association ainsi réglée qui prévaut sur toutes les exploitations sucrières, le partage au tiers brut est préféré au partage par moitié nette [...]. L'association a toutes mes sympathies; c'est de cette voie féconde en généreux résultats que j'espère tirer le perfectionnement des travaux agricoles, l'augmentation des produits, le développement des intelligences par l'émulation. Telle qu'elle est réglée au modèle de contrat que j'ai adopté, elle assure convenablement les deux intérêts engagés; elle est en tout préférable au salaire dont le paiement serait au surplus impossible dans la situation financière actuelle de la colonie [...]», 
Perrinon au Ministre de la Marine et des colonies, Macouba le 10 juillet 1848, ANSOM carton 46, dossier 464.

38. Article $14 \mathrm{du}$ contrat d'association de la Nouvelle Cité, Pierre Dessalles, La vie d'un colon à la Martinique au XIXe siècle. Journal dePierre Dessalles. 1785-1856, Henri de Frémont, Élizabeth Léo, (éds.), Fort-de-France, Désormeaux., 1987 p. 341.

39. Article 2 du contrat d'association de la Nouvelle Cité, Pierre Dessalles, ibid., p. 340.

40. Bilan des tournées de Perrinon [...], Dale Tomich «Contested Terrains. Houses, Provisions Grounds and the Reconstruction of Labour in Post-Emancipation Martinique ", in Mary Turner (éd.) From Chattel Slaves to Wage Slaves, London, Indiana University Press, 1995.

41. Ce qui ne fut acquis dans la métropole qu'en 1848, Robert Baffos, La prud'homie. Son évolution. Paris, Arthur Rousseau, 1908.

42. En Guadeloupe, le procureur-général propose le 10 août 1848 « d'étendre en matière pénale, la compétence du jury cantonal en lui confiant : d'une part, la répression du vagabondage... ; et d'autre part, toutes contraventions possibles du maximum des peines de police... L'autre modification proposée par le même magistrat consisterait à remplacer, comme punition, l'amende par l'envoi à l'atelier de discipline, dans les cas de condamnation pour vagabondage et pour faits tendant à troubler l'ordre ou le travail », Commission coloniale, Première note sur les Jurys cantonaux, janvier 1849.

43. Les jurys cantonaux traitent en effet des accords passés oralement comme ceux écrits. Pour une analyse précise de cette question, voir les travaux de Rebecca Scott, en particulier avec Michael Zeuske, « Property in Writing, Property on the Ground: Pigs, Horses and Citizenship in the Aftermath of Salvery, Cuba, 1880-1909 ", Comparative Studies in Society and History, 44, october 2002, p. 669-699.

44. Afrâio Garcia Jr,. Libres et assujettis. Marché du travail et modes de domination au Nordeste, Paris, Éditions de la Maison des Sciences de l'Homme. 1989, p. 164.

45. Jacques Adélaïde-Merlande, «Les jurys cantonaux de Saint-Pierre, 1848-1851 », Actes du colloque de Saint-Pierre, 1973, p. 50.

46. Au Saint-Esprit, au Marin et aux Anses d'Arlets ce sont les propriétaires qui formulent les demandes (28 mai 1849). Rapport du magistrat délégué à l'inspection des jurys cantonaux de la Martinique.

47. En petit nombre, des conflits personnels sont présentés en jugement, à la Trinité. Eliza, cultivatrice à Sainte-Marie, se plaint de l'usurpation de sa case par Compère Taillis, lui aussi cultivateur. Un groupe de cultivateurs se plaint de Placide, Laguerre et d'autres parce qu'ils ne remplissent pas les clauses du contrat et leur demandent des dommages et intérêts...

48. "Ma tournée du 18 m'a donné la preuve du prestige qu'a le salaire aux yeux du travailleur, avec ce mode, aucune incertitude, aucune discussion, aucun mauvais vouloir ; le but est actuel, il suffit de quelques heures pour l'atteindre, la bonne volonté de chaque jour est stimulée par cet appat puissant. Une préférence extrêmement marquée existe en faveur de ce mode sur celui de l'association dont les résultats plus éloignés, moins déterminés, moins saisissables à l'imagination quoique plus avantageux donnent moins de confiance aux nouveaux libres", Perrinon au Ministre de la Marine et des colonies, Fort-de-France le 19 août 1848.

49. «Le droit de propriété sur les fruits et récoltes pendants par branches et racines acquis aux affranchis en vertu de l'article 2 (« les propriétaires ne pourront priver les affranchis des fruits et récoltes ») du décret du 27 avril sur la répression du vagabondage et de la mendicité doit être limité aux fruits nés avant l'acte d'émancipation, et ne sauraient se perpétuer et s'étendre aux plantations nouvelles. Les motifs de cette décision sont tirés du caractère purement transitoire de la disposition précitée, et des conséquences funestes qu'entraîneraient pour l'agriculture et la libre disposition des terres, la faculté accordée aux travailleurs de revenir indéfiniment sur des habitations qu'ils auraient quittées ou dont ils auraient été renvoyés, pour arracher ou cultiver les produits de leurs anciens jardins ", Première note sur les jurys cantonaux, 31 janvier 1849. 
Voir également Jacques Adélaïde-Merlande, (1973) «Les jurys cantonaux de Saint-Pierre, 1848-1851 », op. cit.

50. Affaire jugée le 19 mai 1849.

51. Ce n'est que deux jours après le jugement, que l'atelier ne vient pas travailler et que, malgré cela, le propriétaire leur donne « la journée qui leur était due »...

52. (28 mai 1849) Rapport du magistrat délégué à l'inspection des jurys cantonaux de la Martinique, p. 83.

53. Tournée du commissaire dans les communes du Nord, 25 juillet 1848.

54. « Avinet a fait l'insolent et le mutin ; je lui ai fait appliquer le fouet (29/11/1839) ; « Joséphine a fait l'insolente; elle voulait quitter le travail plus tôt. Je l'ai fait attacher aux trois piquets » (15/3/1840) ; «Césaire a été insolent et a tenu de mauvais propos, je lui ai fait donner quelques coups de rigoise » $(2 / 3 / 1841)$.

55. Sur toutes les habitations de la Martinique, relate Perrinon, «c'est par l'exactitude au travail que l'on pêchait. Les jours et les heures convenus n'étaient pas donnés complètement par les travailleurs associés. La subordination à un géreur leur répugnait de même que l'assujettissement aux appels ; ils voyaient dans ces formalités des réminiscences de l'esclavage ", Perrinon, 21 octobre 1848.

56. Myriam Cottias, "La séduction coloniale. Damnation et stratégies. Les Antilles, XVII ${ }^{e}-\mathrm{XIX}^{\mathrm{e}}$ siècles ", in Cécile Dauphin, Arlette Farge (éds.), Séduction et Sociétés. Approches historiques, Paris, Le Seuil, 2001.

57. «J'ai appris que M. de Gaalon recherchait la jeune négresse Jeannine : il manquerait à sa promesse, car en arrivant chez moi, il m'a déclaré qu'il prendrait ses maîtresses hors de l'habitation ", La vie d'un colon à la Martinique au XIX' siècle. Journal de Pierre Dessalles. 1785-1856,. 20 avril 1844, op. cit. (supra n. 39).

58. Aucune archive semblable ne semble avoir été conservée, ni pour la Guadeloupe, ni pour la Guyane.

59. (28 mai 1849) Rapport du magistrat délégué à l'inspection des jurys cantonaux de la Martinique, p. 52.

60. Perrinon au Ministre de la Marine et des colonies, Fort-de-France le 19 août1848, carton 46, dossier p. 464.

61. Voir aussi Jacques Adélaïde-Merlande, (1973) «Les jurys cantonaux de Saint-Pierre, 1848-1851 ", op. cit.

62. Lettre du Gouverneur-général au Ministre de la Marine et des colonies, Fort-de-France le 23 octobre 1850 .

63. Pour une discussion de ces termes, voir Claude Meillassoux, Anthropologie de l'esclavage. Le ventre de fer et d'argent, Paris, PUF, 1986 ; Jean-Pierre Sainton, « De l'état d'esclave à "l'état de citoyen". Modalités du passage de l'esclavage à la citoyenneté aux Antilles françaises sous la Seconde République (1848-1850) », Outre-Mers. Revue d'Histoire, 90 (338-339), 2003, p. 47-82.

\section{AUTEUR \\ MYRIAM COTTIAS \\ EHESS/CRH}

